\title{
Multiple temperature model for the information preservation method and its application to nonequilibrium gas flows
}

\author{
Jun Zhang, Jing Fan*, Jianzheng Jiang \\ Laboratory of High Temperature Gas Dynamics, Institute of Mechanics, Chinese Academy of Sciences, Beijing 100190, China
}

\section{A R T I C L E I N F O}

\section{Article history:}

Received 15 January 2011

Received in revised form 18 May 2011

Accepted 22 May 2011

Available online 31 May 2011

\section{Keywords:}

Information preservation method

Direct simulation Monte Carlo method

Multiple temperature model

Nonequilibruim flows

Thermal creep

Rarefied gas dynamics

\begin{abstract}
A B S T R A C T
The information preservation (IP) method has been successfully applied to various nonequilibrium gas flows. Comparing with the direct simulation Monte Carlo (DSMC) method the IP method dramatically reduces the statistical scatter by preserving collective information of simulation molecules. In this paper, a multiple temperature model is proposed to extend the IP method to strongly translational nonequilibrium gas flows. The governing equations for the IP quantities have been derived from the Boltzmann equation based on an assumption that each simulation molecule represents a Gaussian distribution function with a second-order temperature tensor. According to the governing equations, the implementation of IP method is divided into three steps: molecular movement, molecular collision, and update step. With a reasonable multiple temperature collision model and the flux splitting method in the update step, the transport of IP quantities can be accurately modeled. We apply the IP method with the multiple temperature model to shear-driven Couette flow, external force-driven Poiseuille flow and thermal creep flow, respectively. In the former two cases, the separation of different temperature components is clearly observed in the transition regime, and the velocity, temperature and pressure distributions are also well captured. The thermal creep flow, resulting from the presence of temperature gradients along boundary walls, is properly simulated. All of the IP results compare well with the corresponding DSMC results, whereas the IP method uses much smaller sampling sizes than the DSMC method. This paper shows that the IP method with the multiple temperature model is an accurate and efficient tool to simulate strongly translational nonequilibrium gas flows.
\end{abstract}

(c) 2011 Elsevier Inc. All rights reserved.

\section{Introduction}

The transport phenomena, i.e., mass, momentum and heat transfer in different flow regimes, are of great scientific and practical interests. The classification of various flow regimes is based on a dimensionless parameter, i.e., the Knudsen number $(\mathrm{Kn})$, which is defined as the ratio of the mean free path to the characteristic length scale of the system [1]. Typically, the continuum regime is in the range of $\mathrm{Kn} \leqslant 0.01$, the slip regime is $0.01<\mathrm{Kn} \leqslant 0.1$, and the transition regime is $0.1<\mathrm{Kn} \leqslant 10$. In the continuum regime, the Navier-Stokes equations with linear relation between stress and strain and the Fourier's law for heat conduction are adequate to model the gas behavior. In the slip regime, it is generally recognized that the NavierStokes-Fourier equations with slip boundary conditions are capable to accurately simulate gas flows. However, some studies have showed that the Navier-Stokes-Fourier equations give qualitatively incorrect predictions to some certain gas flows in the slip regime, such as the force-driven Poiseuille flow [2]. For gas flows in the transition regime, the Navier-Stokes-Fourier

\footnotetext{
* Corresponding author.

E-mail address: jfan@imech.ac.cn (J. Fan).
} 
equations are known to be inadequate. This is because that the collisions between molecules and collisions of molecules with wall boundaries have the same order of probability, and thus noncontinuum and nonequilibrium effects become important [3]. The transition regime is important for many practical engineering problems, such as the microscale gas flows [4] and hypersonic flow around space vehicles in low earth orbit [5]. Therefore, there is a strong desire and requirement for accurate models that give reliable results in the transition regime.

The fundamental equation describing the molecular nature of gases is the Boltzmann equations, where a single particle distribution function is the primary variable. However, solution of the Boltzmann equation is very difficult due to the term of collision integrals. An alternative approach is to directly model the behavior of the individual molecules for rarefied gas flows. The most popular approach is the direct simulation Monte Carlo (DSMC) method [6]. It has been very successful for solving problems in the field of high-speed rarefied gas flows. Some researchers have applied it to microchannel flows [7-10] and have found it very difficult to obtain statistically convergent results under experimental conditions of interest in micro-electromechanical systems (MEMS). Unlike hypersonic problems, microdevices often work at low Mach numbers, which means that the ratio of macroscopic velocity to thermal velocity is small. Because the statistical scatter inherent in DSMC decreases with the inverse square root of the sample size, an extremely large sample size is required to reduce it to a level that is small in comparison with the macroscopic velocity. This makes DSMC simulation of MEMS flows extremely time-consuming.

Many efforts have been made to reduce the statistical scatter associated with the DSMC method, such as a post-processing technique [11] and the low-variance deviational simulation Monte Carlo method [12,13]. One successful approach is the information preservation (IP) method [14,15]. The IP method was first proposed by Fan and Shen to simulate unidirectional, constant-density, low-speed rarefied gas flows [15]. They proposed to assign each simulation molecule in the DSMC method two velocities. One is the microscopic velocity, which is used to compute molecular motion following the same steps as the DSMC method. The other is called information velocity, which corresponds to the collective velocity of the enormous number of real molecules which are represented by the simulation molecule. Flow velocity is sampled from the information velocity of molecules instead of the microscopic velocity. It has shown that this sampling exhibited much less statistical scatter than regular DSMC sampling for low-speed flows, and thus an IP simulation can save computational time by several orders of magnitude compared with a similar DSMC simulation. Therefore, it is not surprising that many developments have been made to extend the IP method for general flows. For instance, Cai et al. [16] introduced a pressure force term to update the preserved information in 2D isothermal compressible flows, and the flow density was solved using the continuum equation. Later, Shen et al. [17] extended this technique for gas flows in long micro-channels. Stream-wise pressure distributions and mass fluxes through micro-channels given by the IP method agree well with experimental data. For non-isothermal flows, Sun and Boyd [18] proposed to preserve temperature information for molecules. Meanwhile, an additional energy transfer model was proposed to describe the energy flux across an interface. In all these cases, an update step is needed to account for the effects of mass, momentum and energy transport not captured by molecular movements and collisions. In order to accurately update the preserved information, it is very important to establish the theoretical foundation of the IP method. As in Ref. [19], Sun and Boyd established a theoretical frame to update the IP quantities base on the Maxwell transport equation, and suggested two models, namely the local thermal equilibrium (LTE) method and the flux splitting (FS) method, to evaluate the correlation coefficients in the transport equations. The LTE method assumes that each simulation molecule represents a Maxwellian distribution, while FS method splits the molecules into two classes depending on their microscopic velocity and evaluates the correlation coefficients by taking moments of the half-Maxwellian distributions. It has shown that FS model is better to predict the shock structure of normal shock waves and the temperature distributions of thermal Couette flows for all Knudsen number. Recently, Masters and Ye [20] found that it is more reasonable to split the molecules into $2^{\mathrm{N}}$ classes for a problem of $\mathrm{N}$ dimensions, that is, two half spaces in 1-D, four quadrants in 2-D, and eight octants in 3-D. Using the octant FS model, the behavior arising from thermally driven nonequilibrium flows can be well captured.

By far, the IP method has been very successful for simulating various micro-scale gas flows. However, the current IP method has only preserved a single average temperature for each simulation molecule. It is known that there is a kind of strongly nonequilibrium gas flows in which the translational temperature components in various directions may be obviously different. To match the physical reality of these flows, here we propose to preserve multiple translational temperature components in different directions for simulation molecules in the IP method. More important, we derive the transport equations of IP quantities including multiple temperature components based on the Boltzmann equation. A core idea is to assume that each simulation molecule represents a Gaussian distribution with a second-order temperature tensor. Consequently, the IP method can be implemented according to the derived transport equations. This paper is organized as follows: Section 2 provides details on the derivation of the transport equations; Section 3 describes the implementation of the IP method including molecular movement step, molecular collision step and update step. To well capture nonequilibrium effect, we propose a multiple temperature collision model and employ the FS method in the update step. Section 4 concerns the application of the current method to several typical gas flows: shear-driven Couette flow, external force-driven Poiseuille flow and thermal creep flow. The results obtained by IP method are compared with the DSMC results. Finally we present our conclusions in Section 5.

\section{Theoretical foundation of the IP method}

In the DSMC method, each simulation molecule represents a large number of real molecules. The simulation molecules are allowed to move with their microscopic velocity within the computational domain: interacting with boundaries as 
appropriate and with other simulation molecules through a probabilistic treatment of collisions. The macroscopic quantities are obtained by sampling the molecular information. In the IP method, one simulation molecule not only has microscopic velocity, but also has certain preserved information that represents the collective, or macroscopic, properties of a large number of real molecules that could be represented by the simulation molecule. More specifically, the preserved velocity, $\mathbf{U}$, represents the collective or average velocity of these real molecules, i.e., $\mathbf{U}=\overline{\mathbf{c}}$. The preserved temperature, $T$, represents the energy associated with the thermal velocities of these real molecules relative to the preserved velocity, i.e., $T=\left(\overline{\mathbf{c}^{2}}-\mathbf{U}^{2}\right) / 3 R$, where $R$ is the gas constant for the species. The macroscopic quantities can be obtained by sampling the preserved information instead of microscopic information. It has been shown that this sampling exhibits much less statistical scatter than regular DSMC sampling for low-speed flows.

In IP method, the preserved information is carried by simulation molecules. Besides the molecular movements and collisions, other effects may influence the preserved information. For example, the preserved velocity should be updated if there is pressure gradient in the flow field. Therefore, it is necessary to establish the theoretical foundation of IP method. Based on the Maxwell's equation of change, Sun and Boyd [19] obtained the governing equations for the preserved information. Various effects on the transport of preserved information could be described using the corresponding terms in the governing equations, where the so-called "correlation terms" need to be determined by assuming the form of velocity distribution function. Here we directly start from the velocity distribution function to derive the governing equations for the preserved information. Since each simulation molecule represents a large number of real molecules with a distribution function $f$, the preserved velocity $(\mathbf{U})$ and temperature $(T)$ can be defined as the velocity moments of the distribution function, that is,

$$
\begin{aligned}
& \mathbf{U}=\int \mathbf{c} \cdot f d \mathbf{c}, \\
& T=\frac{1}{3 R} \int(\mathbf{c}-\mathbf{U})^{2} \cdot f d \mathbf{c} .
\end{aligned}
$$

Our starting point of derivation is the Boltzmann equation, i.e.,

$$
\frac{\partial(n f)}{\partial t}+\mathbf{c} \cdot \frac{\partial(n f)}{\partial \mathbf{r}}=\left[\frac{\partial(n f)}{\partial t}\right]_{c},
$$

where $n$ is the number density of the group of molecules that are represented by the simulation molecule. The governing equations for the preserved information can be obtained by taking moments of the Boltzmann equation. However, the accurate form of the velocity distribution function is usually unknown. Thus, a valid and reasonable assumption on the distribution function is very crucial for the IP simulations. The simplest assumption is that each simulation molecule represents a Maxwellian distribution based on its preserved velocity $(\mathbf{U})$ and the equilibrium temperature $\left(T_{e q}\right)$, that is,

$$
f=f_{e q}=\frac{1}{\left(2 \pi R T_{e q}\right)^{3 / 2}} \exp \left[-\frac{(\mathbf{c}-\mathbf{U})^{2}}{2 R T_{e q}}\right],
$$

With this velocity distribution function, taking the moments on the Boltzmann equation gives the governing equations as follows:

$$
\begin{aligned}
& \partial_{t} n+\partial_{k}\left(n U_{k}\right)=0, \\
& \partial_{t}\left(n U_{i}\right)+\partial_{k}\left(n U_{i} U_{k}\right)+\partial_{k}\left(n R T_{e q} \delta_{i k}\right)=0, \\
& \partial_{t}\left[n\left(U^{2}+3 R T_{e q}\right)\right]+\partial_{k}\left[n\left(U^{2}+3 R T_{e q}\right) U_{k}\right]+\partial_{k}\left(2 n R T_{e q} U_{k}\right)=0 .
\end{aligned}
$$

For the implementation of IP method, every term in the governing equations is calculated based on statistical average in one computational cell. Eq. (5) is the transport equation of preserved density, which is defined on a cell basis. Eqs. (6) and (7) are the transport equations of preserved velocity and energy, which are defined for every simulation molecule. The second terms on the left hand side of Eqs. (6) and (7) are convection terms, corresponding to the changes due to the microscopic movement of simulation molecules. The third terms on the left hand side of Eqs. (6) and (7) are update terms, which account for the effects of momentum and energy transport not captured by molecular movements. These two terms are similar to the pressure terms in Navier-Stokes equations. That is why pressure force term should be supplied to update the preserved information in the original IP simulations. Note that the Eqs. (6) and (7) is the same as the Eqs. (16) and (17) in the reference [19], where Sun and Boyd obtained the transport equations of IP quantities from Maxwell transport equations. This is because that both of the two derivations are based on the local thermodynamic equilibrium (LTE) assumption, which means that each simulation molecule represents a group of real molecules with a Maxwellian distribution.

However, it is known that the translational temperature components in different directions may have obvious difference in strongly nonequilibrium gas flows. Even if the group of real molecules represented by a simulation molecule is in thermodynamic equilibrium and isotropic state at the initial time, they will become anisotropic in the evolution process 
due to nonequilibrium constraint conditions in the flow field. Therefore, it is more reasonable to assume that each simulation molecule represents a Gaussian distribution with a second-order temperature tensor $\mathbf{T}$, that is,

$$
f=f_{E S}=\frac{1}{[\operatorname{det}(2 \pi R \mathbf{T})]^{1 / 2}} \exp \left[-\frac{1}{2 R}(\mathbf{c}-\mathbf{U}) \cdot \mathbf{T}^{-1} \cdot(\mathbf{c}-\mathbf{U})\right],
$$

where $\mathbf{T}$ is defined as the second-order moments of $f$, i.e.,

$$
T_{i j}=\frac{1}{R} \int\left(c_{i}-U_{i}\right)\left(c_{j}-U_{j}\right) f_{E S} d \mathbf{c} .
$$

It is obvious that $T_{e q}=\left(T_{x x}+T_{y y}+T_{z z}\right) / 3$. Actually, the Gaussian distribution has been used as a middle state to describe the molecular collision process in the early work by Maxwell [21], and then re-discovered by many researchers, such as Holway [22], Levermore [23], Xu and Guo [24]. With Gaussian distribution function, taking the moments on the Boltzmann equation gives the following governing equations:

$$
\begin{aligned}
& \partial_{t} n+\partial_{k}\left(n U_{k}\right)=0, \\
& \partial_{t}\left(n U_{i}\right)+\partial_{k}\left(n U_{i} U_{k}\right)+\partial_{k}\left(n R T_{i k}\right)=\Delta\left(n U_{i}\right), \\
& \left.\partial_{t}\left[n\left(U_{i} U_{j}+R T_{i j}\right)\right]\right)+\partial_{k}\left[n\left(U_{i} U_{j}+R T_{i j}\right) U_{k}\right]+\partial_{k}\left[n R\left(U_{i} T_{j k}+U_{j} T_{k i}\right)\right]=\Delta\left[n\left(U_{i} U_{j}+R T_{i j}\right)\right] .
\end{aligned}
$$

Similar to Eqs. (6) and (7), the second terms on the left hand side of Eqs. (11) and (12) are convection terms, while the third terms on the left hand side of Eqs. (11) and (12) are update terms. There are two obvious advantages in the Eqs. (11) and (12) comparing with Eqs. (6) and (7). First, temperature changes from scalar to second-order tensor. Thus, it is convenient to preserve multiple temperature components in different directions for each simulation molecule in the IP method. The corresponding governing equation for multiple temperature components is the Eq. (12). Second, update terms contain pressure effect as well as shear effect. For example, the update term in Eq. (11) changes from $\partial_{k}\left(n R T_{e q}\right)$ to $\partial_{k}\left(n R T_{i k}\right)$, so it provides the possibility to recover the thermal creep mechanism, which is crucial in thermally driven flows. The terms on the right hand side of Eqs. (11) and (12) are collision terms, which mean that the preserved velocities and temperature components of two collision molecules should be transferred with each other during the process of molecular collision. Due to the implicit expressions of collision terms, it is difficult to solve the Eqs. (11) and (12) mathematically. Fortunately, it is not a problem for the implementation of IP method, where the corresponding convection and collision terms are simulated based on the microscopic molecular movements and collisions, respectively.

\section{Implementation of the IP method}

The IP method is implemented on top of the DSMC method by preserving macroscopic information in simulated molecules. The preserved information does not produce any influence on the motion of molecules and are used only for sampling to obtain the macroscopic field. At the initial time, each simulation molecule is assigned preserved velocity $\mathbf{U}$ and multiple temperatures $\mathbf{T}$ according to the ambient conditions, i.e., $\mathbf{U}=\overline{\mathbf{c}}$ and $T_{x x}=T_{y y}=T_{z z}=T_{e q}$, where $\overline{\mathbf{c}}$ and $T_{e q}$ are the local macroscopic velocity and equilibrium temperature, respectively. The preserved density defined on a cell basis is set the same as ambient density. In every calculating time step, molecular movement, molecular collision and update step are executed to transport the preserved information according to the Eqs. (10)-(12). The movement and collision steps are decoupled as the usual DSMC procedures. The update step must be treated very carefully to capture the nonequilibrium effect. Note that we do not preserve the shear temperature components. This is based on the considerations of two aspects. On the one hand, it is difficult to deal with the distribution of shear temperate components when two molecules collide. Meanwhile, little deviation of shear temperature components may be amplified in the update step, and this would cause unreal results. Thus, it is better to evaluate the shear terms using flux splitting method than directly from the shear temperature components. In the following, we give the details of how to deal with the preserved information in the IP method.

\subsection{Movement step}

Molecules are moved as in the DSMC method, and the preserved information is carried with molecular movements. This step corresponds to perform the convection terms in Eqs. (11) and (12), i.e., $\partial_{k}\left(n U_{i} U_{k}\right)$ and $\partial_{k}\left[n\left(U_{i} U_{j}+R T_{i j}\right) U_{k}\right]$. It should be noted that in the IP method, the preserved information for each simulation molecule is transported according to its microscopic velocity but not the preserved velocity. For one simulation molecule, it corresponds to simulate the terms as $\partial_{k}\left(n U_{i} c_{k}\right)$ and $\partial_{k}\left[n\left(U_{i} U_{j}+R T_{i j}\right) c_{k}\right]$, and the difference between the simulation and the convection terms in Eqs. (11) and (12) is obvious. However, the IP method only requires the correctness of average effect since one simulation molecule represents a large number of real molecules. Consider one computational cell, the average values of preserved velocity and microscopic velocity of molecules are almost the same, that is, $\overline{U_{k}}=\overline{C_{k}}$. Thus, the average effect of the convection terms in one computational 
cell, i.e., $\partial_{k}\left(\overline{n U_{i} U_{k}}\right)$ and $\partial_{k}\left[\overline{n\left(U_{i} U_{j}+R T_{i j}\right) U_{k}}\right]$, can be represented by the movement of DSMC molecules carried with preserved information.

The preserved information of one simulation molecule may be changed when the molecule interacts with a wall boundary. It is reasonable to set the reflected preserved information of the simulation molecule in accordance with the collective behavior of a large number of real molecules represented by the simulation molecule. Namely, if it is a specular reflection, only the normal preserved velocity component will be reserved; if it is a diffuse reflection, the preserved velocity and temperature of the reflected molecules are set the same as the wall velocity and temperature, that is, $\mathbf{U}=\mathbf{U}_{w a l l}$ and $T_{x x}=T_{y y}=T_{z z}=T_{\text {wall }}$.

\subsection{Collision step}

Molecules are selected at random to make pairs, and binary collisions are performed for a subset of these pairs based on the usual DSMC procedures. This step corresponds to perform the collision terms in Eqs. (11) and (12). It is believed that collisions make molecules tend to be in an equilibrium state; hence the preserved information of molecules tends to be the same after collisions. If two molecules collide, a simple collision model can be derived based on the conservation of momentum and energy, that is,

$$
\begin{aligned}
& U_{i, 1}^{*}=U_{i, 2}^{*}=0.5\left(U_{i, 1}+U_{i, 2}\right), \\
& T_{i i, 1}^{*}=T_{i i, 2}^{*}=\left(T_{i i, 1}+T_{i i, 2}\right) / 3+\sum_{j \neq i}\left(T_{j j, 1}+T_{j j, 2}\right) / 12+\sum_{j=1}^{3}\left(U_{j, 1}-U_{j, 2}\right)^{2} /(12 R),
\end{aligned}
$$

where the subscript $i$ represents the direction, the subscript 1 and 2 represent the index of the two collision molecules, and the symbols with superscript $*$ represent the post-collision quantities. Note that $T_{i i}$ represents the diagonal component of temperature tensor. For example, Eq. (14) for $T_{x x}$ yields:

$$
T_{x x, 1}^{*}=T_{x x, 2}^{*}=\left(T_{x x, 1}+T_{x x, 2}\right) / 3+\left(T_{y y, 1}+T_{y y, 2}\right) / 12+\left(T_{z z, 1}+T_{z z, 2}\right) / 12+\sum_{j=1}^{3}\left(U_{j, 1}-U_{j, 2}\right)^{2} /(12 R) .
$$

However, numerical tests show that the aforementioned simple collision model cannot correctly simulate the viscosity and thermal conductivity of the flows. Hence, the simple collision model needs to be modified. The preserved macroscopic information for two collision molecules may be different after one collision. As described in reference [18], it may depend on the relative speed of the two molecules, the deflection angle in the collision plane, and so no. Sun and Boyd [18] proposed a phenomenological model for the distribution of the preserved velocity and average temperature for the two molecules as follows:

$$
\begin{aligned}
& U_{i, 1}^{*}=0.5\left(U_{i, 1}+U_{i, 2}\right)+0.5 c_{\mu} \cos (\theta)\left(U_{i, 1}-U_{i, 2}\right), \\
& U_{i, 2}^{*}=0.5\left(U_{i, 1}+U_{i, 2}\right)-0.5 c_{\mu} \cos (\theta)\left(U_{i, 1}-U_{i, 2}\right), \\
& T_{1}^{*}=0.5\left(T_{1}+T_{2}\right)+0.5 c_{\lambda} \cos (\theta)\left(T_{1}-T_{2}\right)+\left(1-c_{\mu}^{2} \cos ^{2} \theta\right) \sum_{j=1}^{3}\left(U_{j, 1}-U_{j, 2}\right)^{2} /(12 R), \\
& T_{2}^{*}=0.5\left(T_{1}+T_{2}\right)-0.5 c_{\lambda} \cos (\theta)\left(T_{1}-T_{2}\right)+\left(1-c_{\mu}^{2} \cos ^{2} \theta\right) \sum_{j=1}^{3}\left(U_{j, 1}-U_{j, 2}\right)^{2} /(12 R),
\end{aligned}
$$

where $c_{\mu}$ and $c_{\lambda}$ are assumed to be constants depending on gas species, and $\theta$ is defined as the deflection angle in the collision plane, i.e.,

$$
\cos \theta=\left(\sum_{i=1}^{3}\left(c_{i, 1}-c_{i, 2}\right)\left(c_{i, 1}^{*}-c_{i, 2}^{*}\right)\right) /\left(v_{r} \cdot v_{r}^{*}\right)
$$

where $v_{r}$ and $v_{r}^{*}$ are the relative speed of the two molecules before and after collision, respectively. Here we employ the same model as that proposed by Sun and Boyd for the distribution of preserved velocity, but for the preserved temperature, some revisions should be made because multiple temperature model is used here. Consider the variance of preserved temperature component in a certain direction, the effect of the deflection angle component in the parallel direction is usually different from the effects of the deflection angle components in the two other vertical directions. Thus, we introduce two parameters $c_{\lambda 1}$ and $c_{\lambda 2}$ in the model of distribution of preserved temperature components as follows: 


$$
\begin{aligned}
T_{i i, 1}^{*}= & 0.5\left(T_{\alpha, 1}+T_{\alpha, 2}\right)+0.5 c_{\lambda, 1} \cos \left(\theta_{i}\right) \cos \left(\theta_{i}^{*}\right)\left(T_{\alpha, 1}-T_{\alpha, 2}\right)+\sum_{j \neq i} 0.5 c_{\lambda, 2} \cos \left(\theta_{j}\right) \cos \left(\theta_{j}^{*}\right)\left(T_{\alpha, 1}-T_{\alpha, 2}\right) \\
& +\left(1-c_{\mu}^{2} \cos ^{2} \theta\right) \sum_{j=1}^{3}\left(U_{j, 1}-U_{j, 2}\right)^{2} /(12 R), \\
T_{i i, 2}^{*}= & 0.5\left(T_{\alpha, 1}+T_{\alpha, 2}\right)-0.5 c_{\lambda, 1} \cos \left(\theta_{i}\right) \cos \left(\theta_{i}^{*}\right)\left(T_{\alpha, 1}-T_{\alpha, 2}\right)-\sum_{j \neq i} 0.5 c_{\lambda, 2} \cos \left(\theta_{j}\right) \cos \left(\theta_{j}^{*}\right)\left(T_{\alpha, 1}-T_{\alpha, 2}\right) \\
& +\left(1-c_{\mu}^{2} \cos ^{2} \theta\right) \sum_{j=1}^{3}\left(U_{j, 1}-U_{j, 2}\right)^{2} /(12 R),
\end{aligned}
$$

where $\cos \theta_{i}=\left(c_{i, 1}-c_{i, 2}\right) / v_{r}, \cos \theta_{i}^{*}=\left(c_{i, 1}^{*}-c_{i, 2}^{*}\right) / v_{r}^{*}$, and $T_{\alpha, n}=2 T_{i, i, n} / 3+\sum_{j \neq i} T_{j j, n} / 6$. As in reference [18], the values of $c_{\mu}$ is determined when the numerical shear tress agrees with the theoretical result and experimental data in low speed Couette flows, while $c_{\lambda 1}$ and $c_{\lambda 2}$ are determined when the numerical heat flux agrees with the DSMC result in thermal Couette flows. For Argon gas, we determined the three constants: $c_{\mu}=-0.18, c_{\lambda 1}=0.80$ and $c_{\lambda 2}=1.56$. In Section 4 , the determined constants will be used in the simulation of flows with Argon gas.

\subsection{Update step}

Besides the molecular movements and collisions, the preserved information should be modified according to the update terms in Eqs. (10)-(12). Based on the average over all molecules in each computational cell, the governing equations for update can be written as follows:

$$
\begin{aligned}
& \partial_{t} n=-\partial_{k}\left(n \overline{U_{k}}\right), \\
& \partial_{t}\left(n \overline{U_{i}}\right)=-\partial_{k}\left(n R \overline{T_{i k}}\right), \\
& \left.\partial_{t}\left[n\left(\overline{U_{i} U_{j}+R T_{i j}}\right)\right]\right)=-\partial_{k}\left[n R\left(\overline{U_{i} T_{j k}+U_{j} T_{k i}}\right)\right] .
\end{aligned}
$$

Note that the update terms in Eqs. (24), (25) are obtained by integrating the Gaussian distribution over the whole-space, for example,

$$
R \overline{T_{i k}}=\overline{\int_{-\infty}^{+\infty} \int_{-\infty}^{+\infty} \int_{-\infty}^{+\infty}\left(c_{i}-U_{i}\right)\left(c_{k}-U_{k}\right) f_{E S} d c_{i} d c_{j} d c_{k}}
$$

In strongly nonequilibrium flows, the preserved information carried by molecules with inverse movement directions may be very different. Thus, a more accurate evaluation of the update terms is to include certain nonequilibrium characteristic of the flow. Here we employ the idea of flux splitting method, which is proposed by Sun and Boyd [19] and is developed by Masters and Ye [20]. In each computational cell, molecules are classified into different classes according to their microscopic velocities, and the complete update terms are obtained by summing the average contribution from each class. For each simulation molecule, the contribution to its class is evaluated by integrating the Gaussian distribution function over the half-space determined by comparing its microscopic velocity $\left(c_{i}\right)$ to its preserved velocity $\left(U_{i}\right)$. In this way, if considering the nonequilibrium characteristic, it is more reasonable to evaluate the term $R \overline{T_{i k}}$ for one-dimensional flows as follows:

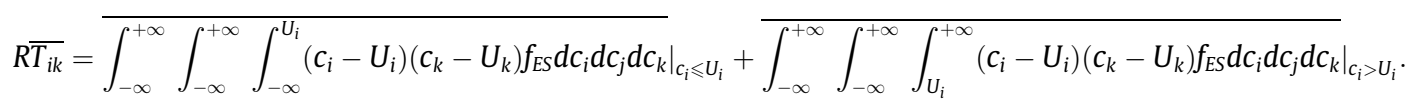

For two-dimensional flows, the term $R \overline{T_{i k}}$ could be evaluated by summing the contributions from four classes as follows:

$$
\begin{aligned}
R \overline{T_{i k}} & =\left.\overline{\int_{-\infty}^{+\infty} \int_{-\infty}^{U_{j}} \int_{-\infty}^{U_{i}}\left(c_{i}-U_{i}\right)\left(c_{k}-U_{k}\right) f_{E S} d c_{i} d c_{j} d c_{k}}\right|_{\left(c_{i} \leqslant U_{i}, c_{j} \leqslant U_{j}\right)}+\frac{\left.\overline{\int_{-\infty}^{+\infty} \int_{-\infty}^{U_{j}} \int_{U_{i}}^{+\infty}\left(c_{i}-U_{i}\right)\left(c_{k}-U_{k}\right) f_{E S} d c_{i} d c_{j} d c_{k}}\right|_{\left(c_{i}>U_{i}, c_{j} \leqslant U_{j}\right)}}{} \\
& +\left.\int_{-\infty}^{+\infty} \int_{U_{j}}^{+\infty} \int_{-\infty}^{U_{i}}\left(c_{i}-U_{i}\right)\left(c_{k}-U_{k}\right) f_{E S} d c_{i} d c_{j} d c_{k}\right|_{\left(c_{i} \leqslant U_{i}, c_{j}>U_{j}\right)}+\left.\int_{-\infty}^{+\infty} \int_{U_{j}}^{+\infty} \int_{U_{i}}^{+\infty}\left(c_{i}-U_{i}\right)\left(c_{k}-U_{k}\right) f_{E S} d c_{i} d c_{j} d c_{k}\right|_{\left(c_{i}>U_{i}, c_{j}>U_{j}\right)} .
\end{aligned}
$$

With flux splitting method, the shear mechanism can be recovered although we don't preserve shear temperature components directly. By similar means, the term $R\left(\overline{U_{i} T_{j k}+U_{j} T_{k i}}\right)$ can be derived. For one-dimensional flows, the term $R \overline{U_{i} T_{j k}}$ could be expressed as follows:

$$
\begin{aligned}
& R \overline{U_{i} T_{j k}}=\left.\overline{\overline{c_{i}} \cdot \int_{-\infty}^{+\infty} \int_{-\infty}^{+\infty} \int_{-\infty}^{U_{i}}\left(c_{j}-U_{j}\right)\left(c_{k}-U_{k}\right) f_{E S} d c_{i} d c_{j} d c_{k}}\right|_{c_{i} \leqslant U_{i}} \\
& +\left.\overline{c_{i}} \cdot \int_{-\infty}^{+\infty} \int_{-\infty}^{+\infty} \int_{U_{i}}^{+\infty}\left(c_{j}-U_{j}\right)\left(c_{k}-U_{k}\right) f_{E S} d c_{i} d c_{j} d c_{k}\right|_{c_{i}>U_{i}} .
\end{aligned}
$$




\subsection{Sampling}

The flow properties are obtained by sampling preserved information of molecules in each computational cell. The flow velocity is,

$$
\mathbf{U}_{c}=\overline{\mathbf{U}} \text {. }
$$

The temperature component is,

$$
\begin{aligned}
& T_{x x, c}=\overline{T_{x x}}+\frac{1}{R}\left(\overline{U_{x}^{2}}-{\overline{U_{x}}}^{2}\right), \\
& T_{y y, c}=\overline{T_{y y}}+\frac{1}{R}\left(\overline{U_{y}^{2}}-{\overline{U_{y}}}^{2}\right), \\
& T_{z z, c}=\overline{T_{z z}}+\frac{1}{R}\left(\overline{U_{z}^{2}}-{\overline{U_{z}}}^{2}\right),
\end{aligned}
$$

and the average temperature is

$$
T_{c}=\left(T_{x x, c}+T_{y y, c}+T_{z z, c}\right) / 3 .
$$

The flow number density $n_{c}$ can be obtained by using time or ensemble averaging of the preserved density defined on a cell basis.

\subsection{Summary of the IP procedure}

At the initial time, every simulation molecule is given a preserved velocity and multiple temperatures according to the ambient conditions. The preserved density defined on a cell basis is set the same as the ambient density. In each calculating time step, the transport of preserved information includes molecular movement, molecular collision and update steps. The detailed procedure is summarized as follows:

(a) Molecules move according to their microscopic velocities as in the DSMC method, and the preserved information is carried with the molecular movements. In this step, the preserved information of each simulation molecule keeps invariant.

(b) Collision pairs are determined by the DSMC collision scheme. If two molecules are selected as a collision pair, their preserved velocities will be changed according to Eqs. (16) and (17), and their preserved temperature components will be changed according to Eqs. (21) and (22).

(c) After molecule movements and collisions, preserved information are updated following the Eqs. (23)-(25). The update terms on the right hand side of governing equations are obtained based on the average over all molecules in each computational cell as formulas defined in Section 3.3. In addition, it is assumed that the update terms apply equally to individual molecules in each computational cell. In this way, the preserved density of each computational cell and preserved information of each simulation molecule can be updated according to Eqs. (23)-(25).

Steps (a)-(c) are repeated in the process of calculation. For steady flows, time averaging is used to obtain the final results after the flow reaches a steady state. For unsteady flows, repeat steps (a)-(c) for the desired sampling size, and ensemble averaging is used to obtain the instantaneous flow field.

\section{Numerical experiments}

In previous studies, the IP method has exhibited the ability to reduce the statistical scatter in the DSMC method. In the present paper, we employ the IP method with multiple temperature model to simulate three typical gas flows: shear-driven Couette flow, external force-driven Poiseuille flow and thermal creep flow. In the former two cases, we test the capability of IP method to capture the different temperature components in strongly translational nonequilibrium flows. In thermal creep flow, we test the capability of IP method to reproduce the creep mechanism.

\subsection{Shear-driven Couette flow}

Shear-driven Couette flow is popularly encountered in micromotors, comb mechanisms, and microbearings. In the simplest case, the Couette flow can be used as a prototype flow to model such flows driven by a moving plate. In the continuum regime, accurate solutions can be obtained by solving Navier-Stokes equations. However, in the transition regime, it is not easy to obtain accurate solutions due to the rarefied gas effect. Meanwhile, there are few experimental data and simulation results in the literature. Hence, the DSMC results are used to compare with the IP results.

Fig. 1 shows the schematic diagram of the Couette flow. The two infinite parallel plates are separated by a distance L. The upper plate is moving with fixed speed, and the lower plate is quiescent. In our IP and DSMC simulations, the working gas is 


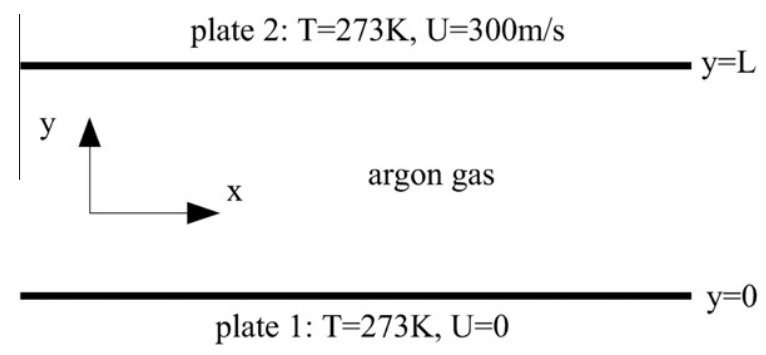

Fig. 1. Schematic diagram for the Couette flow.

Argon. The temperature of both plates is $273 \mathrm{~K}$, and the velocity of upper plate is $300 \mathrm{~m} / \mathrm{s}$. The use of this wall velocity is from the consideration of two aspects. First, it is easy for DSMC to reduce the statistical scatter to an acceptable level. Second, because of the large velocity gradient, the dissipation causes the gas temperature to increase significantly. Thus, it is convenient to check the multiple temperature model for IP method proposed here. The one-dimensional computational domain is divided into cells within which collision pairs are selected. The size of cells is smaller than $0.5 \lambda$, and the computational time step equals to $0.1 \tau$, where $\lambda$ and $\tau$ are the molecular mean free path and mean collision time, respectively. The macroscopic quantities are obtained by sampling the preserved information for IP method or by sampling the microscopic information for DSMC method.

The preserved information is carried by molecules with movements. Diffusive reflections are assumed at both of plates. It means that the molecules colliding with these walls rebound with the same macroscopic or preserved velocity and temperature as that of the walls, while the thermal velocities of molecules are sampled from half-range Maxwellian distribution at the temperature of the corresponding wall. Variable hard sphere (VHS) model is employed to describe the interaction of molecular collisions. For IP method, collision pairs are selected based on the usual DSMC procedure. The post-collision preserved velocities are distributed according to Eqs. (16) and (17), and the post-collision preserved temperature components are distributed according to Eqs. (21) and (22).

Besides movement and collision steps, update step is needed for IP method based on the analysis in Section 3 . For onedimensional Couette flow, gradient of macroscopic flow quantities only exists in the y direction, hence the governing equations can be simplified as:

$$
\begin{aligned}
& \frac{\partial\left(n \overline{U_{y}}\right)}{\partial t}=-\frac{\partial\left(n R \overline{T_{y y}}\right)}{\partial y} \\
& \frac{\partial\left(n \overline{\left(U_{y}^{2}+R T_{y y}\right)}\right)}{\partial t}=-\frac{\partial\left(2 n R \overline{U_{y} T_{y y}}\right)}{\partial y} .
\end{aligned}
$$

Considering the nonequilibrium characteristic in the y direction, each simulation molecule is classified as belonging to one class based on its microscopic velocity $\left(c_{y}\right)$ relative to its preserved velocity $\left(U_{y}\right)$. According to the Eqs. (27) and (29), the update terms can be evaluated as:

$$
\begin{aligned}
& \overline{T_{y y}}=\left.\overline{\frac{1}{2} T_{y y}}\right|_{c_{y} \leqslant U_{y}}+\left.\overline{\frac{1}{2} T_{y y}}\right|_{c_{y}>U_{y}}, \\
& \overline{U_{y} T_{y y}}=\left.\frac{1}{2} T_{y y}\left(U_{y}-\sqrt{\frac{R T_{y y}}{2 \pi}}\right)\right|_{c_{y} \leqslant U_{y}}+\left.\overline{\frac{1}{2} T_{y y}\left(U_{y}+\sqrt{\frac{R T_{y y}}{2 \pi}}\right)}\right|_{c_{y}>U_{y}} .
\end{aligned}
$$

Note that the expression of Eq. (38) is similar to Eq. (24) in reference [19], where the flux splitting method is used to calculating the update term. The difference between them is that the latter is based on the total energy, and here we clearly show that the energy update step should be particularly implemented on the energy component in the $y$ direction. This can be explained based on gas kinetic theory as follows. When one molecule moves across an interface vertical to the $y$ direction, the average translational energy component in the $y$ direction carried by the molecule is $k T$ whereas the corresponding volume average is only $\frac{1}{2} k T$, while both of the average translational energy components in the $x$ and $z$ directions carried by the molecule are the same as the corresponding volume averages, i.e., $\frac{1}{2} k T$. Therefore, the update should be performed on the energy component in the $y$ direction.

In the following, simulation results of Couette flow ranging from continuum regime to transition regime are given. Fig. 2 shows the velocity and temperature distributions along the $\mathrm{y}$ direction for $\mathrm{Kn}=0.01$, where the solid lines are the current IP results and circles are the DSMC results. Actually, three temperature components are plotted for both IP and DSMC results, even though they are indistinguishable. At this Knudsen number, the separation between the temperatures is too small to be seen. This is because that there are enough molecular collisions to make the translational energy equally distribute in the three directions. To quantify the level of agreement between the IP and DSMC results, we use the normalized root mean square 

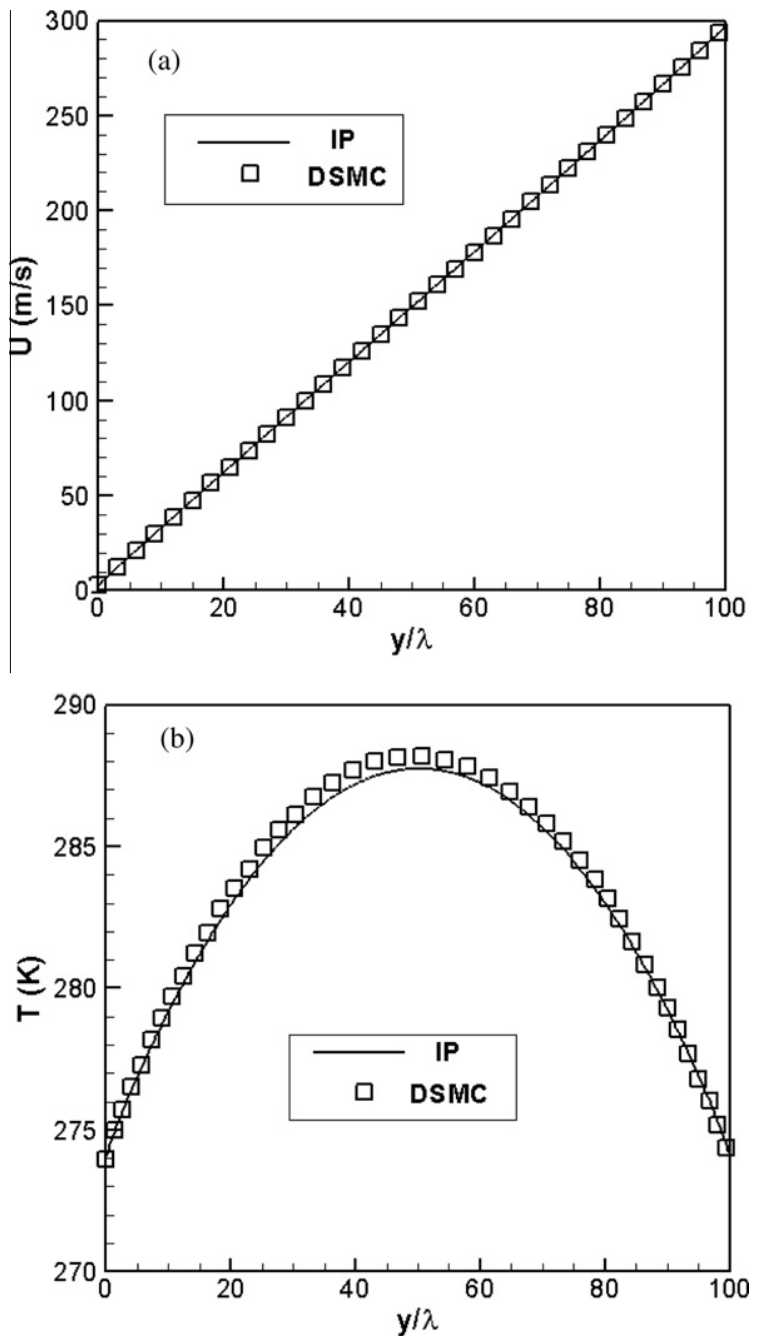

Fig. 2. Velocity (a) and temperature (b) distributions in Couette flow for $\mathrm{Kn}=0.01$.

deviation (RMSD) to compare differences between the IP and DSMC results. For Couette flow at $\mathrm{Kn}=0.01$, the normalized RMSD of $U$ and $T$ are $0.17 \%$ and $0.12 \%$, respectively. It shows that the IP and DSMC results compare well with each other. As the Knudsen number increases to 0.1, the three temperatures can be clearly observed in Fig. 3, where the magnitudes of the temperature are distributed from the highest $T_{x x}$, to $T_{z z}$, and to the lowest $T_{y y}$. At $\mathrm{Kn}=0.1$, both velocity and temperature distributions obtained by IP method have a fair agreement with the DSMC results. The normalized RMSD of $U, T, T_{x x}, T_{y y}$ and $T_{z z}$ obtained by IP and DSMC methods are $0.17 \%, 0.13 \%, 0.25 \%, 0.09 \%$ and $0.06 \%$, respectively. As the Knudsen number increases to 0.5 , both velocity and temperature distributions shown in Fig. 4 become flat in comparison with that under the condition of small Knudsen numbers because the velocity slip and temperature jump at the wall boundaries are very large. In this case, the normalized RMSD of $U, T, T_{x x}, T_{y y}$ and $T_{z z}$ obtained by IP and DSMC methods are $0.29 \%, 0.12 \%, 0.73 \%, 0.25 \%$ and $0.37 \%$, respectively. It is shown that the discrepancies between the temperature components obtained by IP and DSMC method become larger as the increasing of Knudsen number. This is because that there are three constants in the present IP collision model. As described in Section 3.2, they are determined when the shear stress and heat flux predicted by IP method agree with the experimental and DSMC results in Couette flows for $\mathrm{Kn}=0.01$. Therefore, it is not surprising that the discrepancy between IP and DSMC results becomes larger as the increasing of Knudsen number. In the future, a more general collision model could be developed by considering the effect of Knudsen number. Note that the IP method requires more computer memory to preserve macroscopic information and spends extra time in calculating the transport of preserved information. However, the advantage of the IP method is that it needs much less sampling sizes comparing with the DSMC method. For example, in the case of $\mathrm{Kn}=0.1$, the DSMC method requires about $2.4 \times 10^{7}$ sampling molecules per computational cell to get smooth distributions of temperature components, while the IP method only needs about $2.4 \times 10^{4}$ sampling molecules per computational cell to 

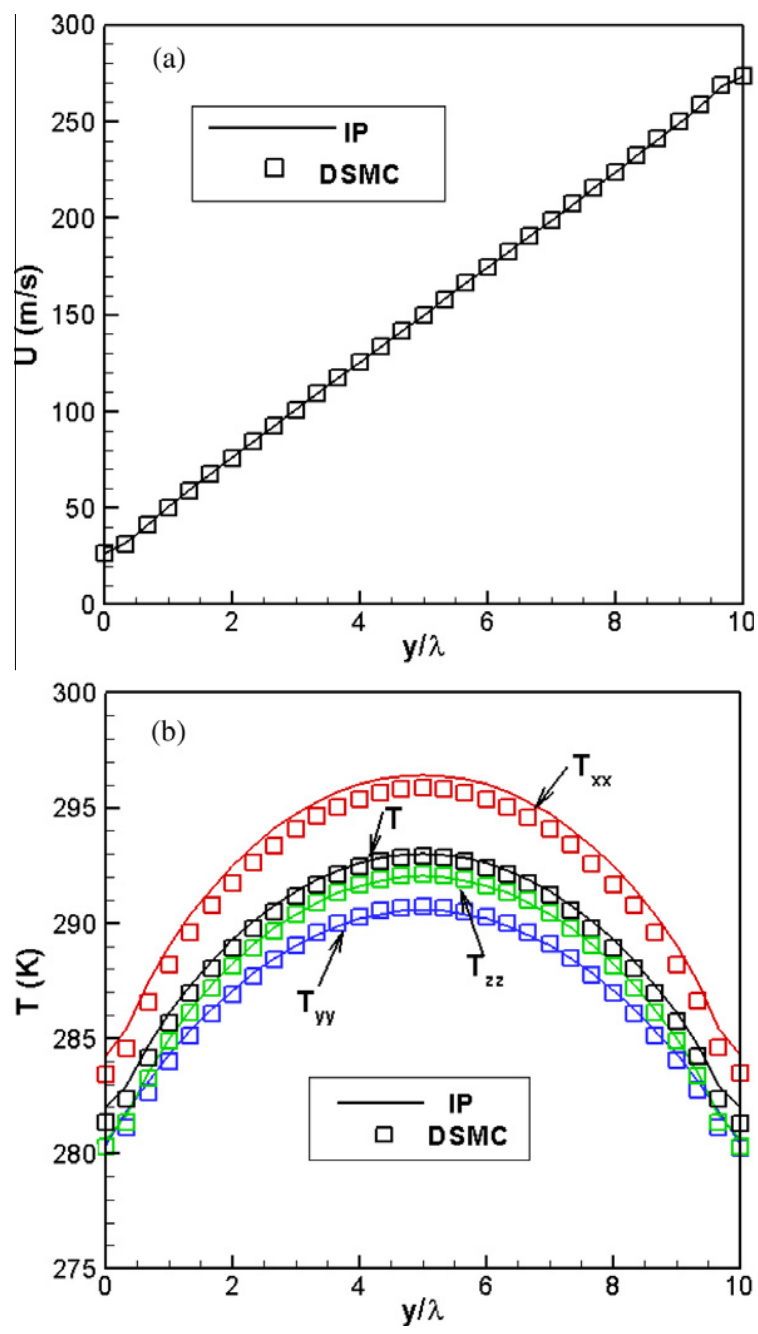

Fig. 3. Velocity (a) and temperature (b) distributions in Couette flow for $\mathrm{Kn}=0.1$.

obtain the same level of results. Consequently, the total computation time for IP method is only about $0.5 \%$ of that for DSMC method.

\subsection{External force-driven Poiseuille flow}

Another typical unidirectional flow is the external force-driven Poiseuille flow, which is confined between two stationary infinite parallel plates and is driven by the uniform external force in the x direction, i.e., in the direction parallel to the plates. Fig. 5 gives the schematic diagram of the Poiseuille flow. If one considers this problem on the basis of the Navier-Stokes equations, it is a simple unidirectional flow. It is generally recognized that in the slip flow regime, the Navier-Stokes equations with the slip boundary condition are capable to accurately simulate the gas flow. However, various studies have showed that some important phenomena in the external force-driven Poiseuille flow in the slip regime cannot be obtained by the Navier-Stokes equation. One of such phenomena is a bimodal shape of the temperature profile with a slight hollow at the center between the plates. This effect was first pointed out by Mansour et. al [2] on the basis of numerical results obtained by the DSMC method and of an explicit perturbation solution derived earlier by Tij and Santos [25] using the Bhatnagar-Gross-Krook (BGK) model. Recently, Xu et. al [26] well reproduced the effect by using an extended BGK model with multiple translational temperature. Here we employ the IP method to simulate Poiseuille flow and compare the IP results with the DSMC results.

In our simulations, the working gas is Argon, and the temperature of the two parallel plates is kept at $273 \mathrm{~K}$. The implementation of the IP and DSMC method for Poiseuille flow is the same as that described in the Section 4.1 for shear-driven Couette flow except that an velocity increment $a \Delta t$ should be added to both of the microscopic velocity and preserved velocity for each simulated molecule during a calculating time step, where $a$ is the acceleration caused by the external force. Fig. 6 

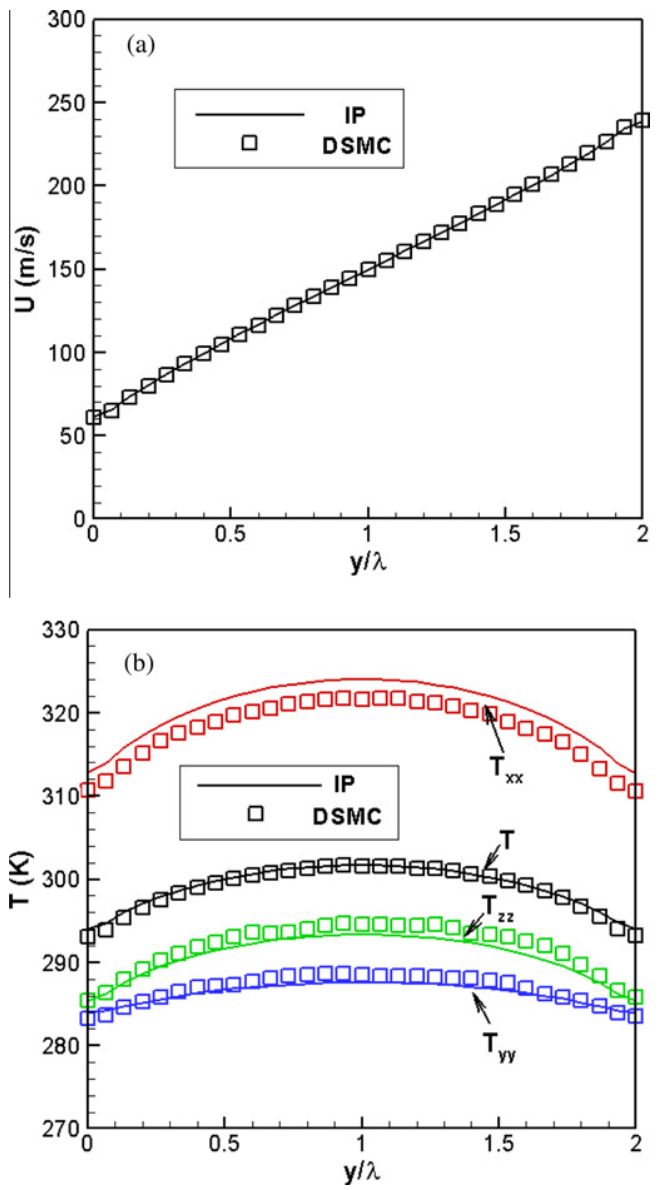

Fig. 4. Velocity (a) and temperature (b) distributions in Couette flow for $\mathrm{Kn}=0.5$.

plate 2: $\mathrm{T}=273 \mathrm{~K}, \mathrm{U}=0$

$y=L$

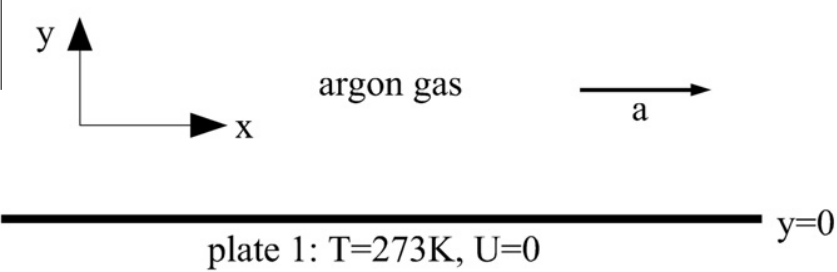

Fig. 5. Schematic diagram for the Poiseuille flow.

shows the velocity and temperature distributions along the $\mathrm{y}$ direction for $\mathrm{Kn}=0.01$ and $a=3.52 \times 10^{8} \mathrm{~m} / \mathrm{s}^{2}$. The simulation results are close to the solution obtained by Navier-Stokes equations. At this Knudsen number, the difference between the temperature components is indistinguishable. Fig. 7 shows the velocity and temperature profiles along the y direction for $\mathrm{Kn}=0.1$ and $a=3.52 \times 10^{10} \mathrm{~m} / \mathrm{s}^{2}$. The velocity distribution is similar to the case for $\mathrm{Kn}=0.01$ except for a larger slip velocity at the boundary, but the three temperature components are obviously separated. The temperature minimum in both $T_{x x}$ and the average temperature $T$ can be clearly observed in Fig. 7(b). Meanwhile, the curved pressure distribution is well captured as shown in Fig. 8. All of the IP results and DSMC results have a good agreement. The advantage of IP method is that it uses much smaller sampling sizes than DSMC method. For example, in the case of $\mathrm{Kn}=0.1$, the DSMC method requires about $4.8 \times 10^{7}$ sampling molecules per computational cell to accurately show the temperature and pressure distributions, while the IP method only needs about $4.8 \times 10^{4}$ sampling molecules per computational cell to obtain the same level of results. The total computation time for IP method is about $0.5 \%$ of that for DSMC method. 

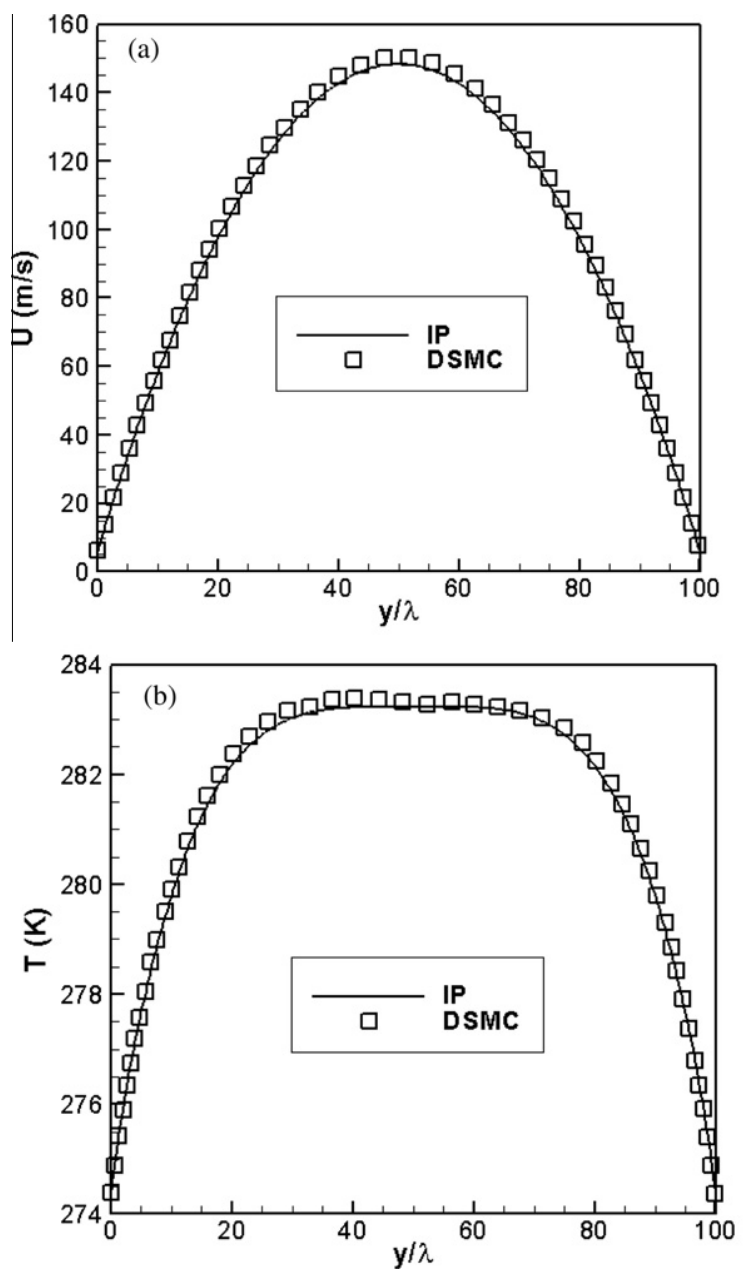

Fig. 6. Velocity (a) and temperature (b) distributions in Poiseuille flow for $\mathrm{Kn}=0.01$.

\subsection{Thermal creep flows}

The thermal creep flow, resulting from the presence of temperature gradients along boundary walls, was first discovered by Reynolds and later studied by Maxwell. By far, a number of microscale and nanoscale systems have utilized this phenomenon for pumping and propulsion [27]. The creep mechanism can be explained on the basis of kinetic theory as follows. Consider a gas with a temperature gradient parallel to a confining wall, the average tangential momentum of molecules leaving the wall from a given point is zero while the molecules arriving from hot regions impart more tangential momentum to the wall than molecules arriving from colder regions. Thus, a shear stress is exerted on the wall, with the gas flowing from the colder to the hotter region as a reaction force. In order to know the detailed flow field of thermal creep flows, many different approaches have been proposed, including various solutions of the linearized Boltzmann transport equation [28,29], near continuum slip models [30], and DSMC simulations [31]. Linearized BTE methods are capable to simulate gas flows with small thermal gradient, i.e., weakly nonequilibrium, but are likely inadequate for the large thermal gradients that may be encountered in microscale and nanoscale systems. Near continuum models are only applicable for a small range of flow conditions. Although DSMC method has been used to simulate thermal creep flows in the whole regime very successfully, the statistical scatter makes the application of DSMC method to low-speed flows is very expensive. This situation has motivated the development of specialized DSMC method. Recently, Master and Ye [20] simulated thermal creep flows by using IP method, where the Maxwell transport equation is used to update the preserved information and flux splitting model is developed to evaluate the correlation terms in the transport equations. Their simulations showed that IP method is able to accurately model the behavior arising from thermally driven nonequilibrium flows with much less sample sizes than DSMC method. Here we employ the multiple temperature model for IP method to simulate thermal creep flows. Eqs. (10)-(12) are used to transport the preserved information of molecules. Comparing with the Maxwell transport equation used by Masters and Ye, it is obvious that the term $\partial_{k}\left(n R T_{i k}\right)$ in Eq. (11) provides the potential creep effect with the shear temperature 

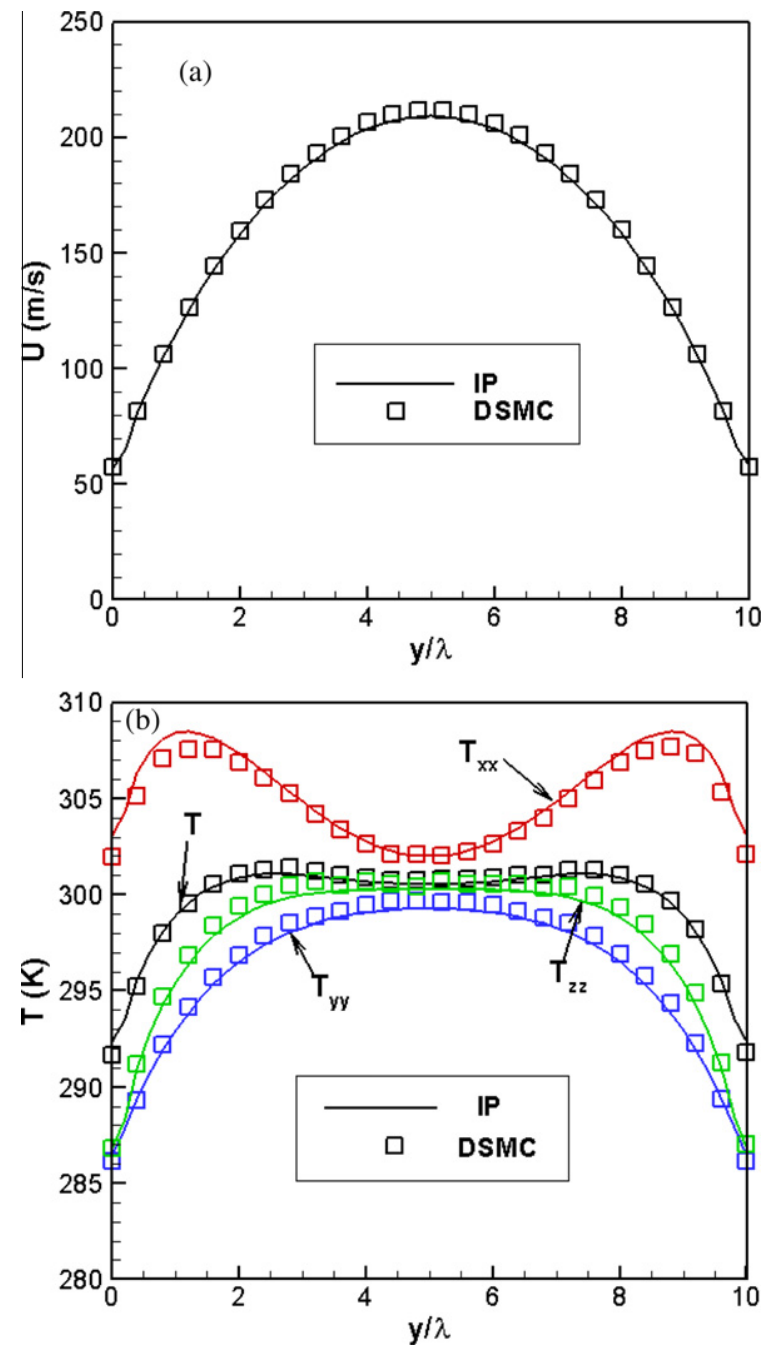

Fig. 7. Velocity (a) and temperature (b) distributions in Poiseuille flow for $\mathrm{Kn}=0.1$.

components. Although the shear temperature components are not preserved for molecules in IP method, the shear stress effect could be obtained in the term by using the idea of flux splitting.

We consider a two-dimensional model as shown in Fig. 9. The two vertical walls of the channel are maintained at two different temperatures $T_{1}=273 \mathrm{~K}$ and $T_{2}=573 \mathrm{~K}$. The temperature of the two horizontal walls varies linearly along the length of the channel. The working gas (Argon) is initially in thermal equilibrium with the walls, i.e., $T(x, y)=\left(T_{2}-T_{1}\right) x /$ $L+T_{1}$, and at a uniform pressure of one atmosphere, i.e., $P(x, y)=1 \mathrm{~atm}$. The two-dimensional computational domain is divided into 200 cells along the length and 40 cells along the width. Diffusive reflections are assumed at walls. Variable hard sphere (VHS) model is employed to describe the interaction of molecular collisions. The movement and collision steps are performed as described in Section 3. For two-dimensional gas flows, the governing equation for update step could be written as:

$$
\begin{aligned}
& \frac{\partial\left(n \overline{U_{x}}\right)}{\partial t}=-\frac{\partial\left(n R \overline{T_{x x}}\right)}{\partial x}-\frac{\partial\left(n R \overline{T_{x y}}\right)}{\partial y}, \\
& \frac{\left.\partial n \overline{U_{y}}\right)}{\partial t}=-\frac{\partial\left(n R \overline{T_{x y}}\right)}{\partial x}-\frac{\left.\partial n R \overline{T_{y y}}\right)}{\partial y}, \\
& \frac{\left.\partial n \overline{\left(U_{x}^{2}+R T_{x x}\right)}\right)}{\partial t}=-\frac{\left.\partial 2 n R \overline{U_{x} T_{x x}}\right)}{\partial x}-\frac{\left.\partial 2 n R \overline{U_{x} T_{x y}}\right)}{\partial y},
\end{aligned}
$$




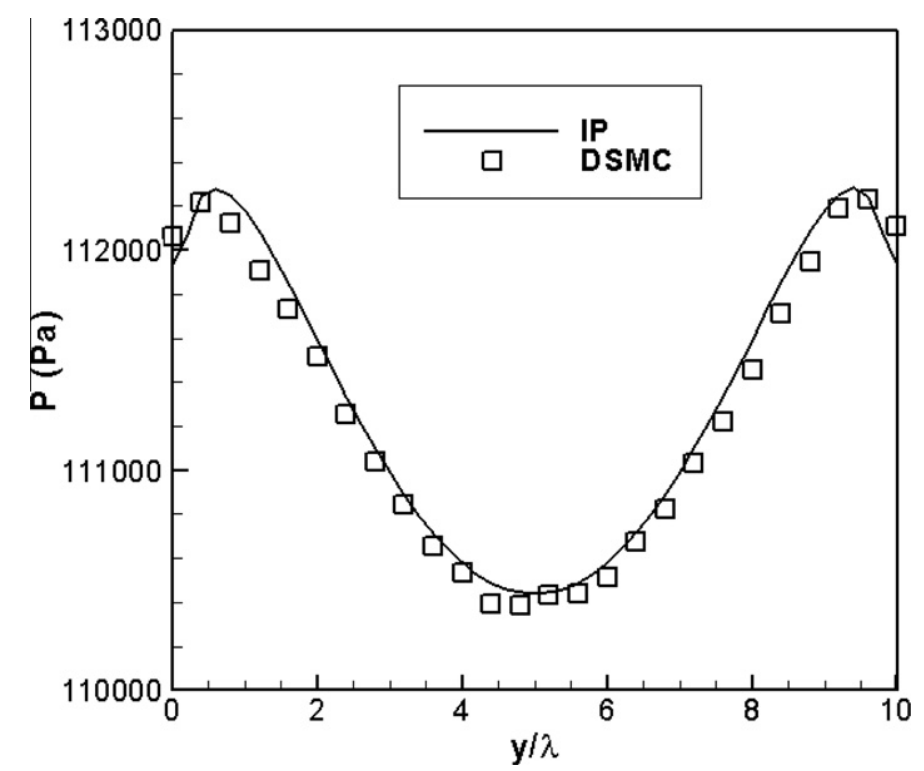

Fig. 8. Pressure distribution in Poiseuille flow for $\mathrm{Kn}=0.1$.

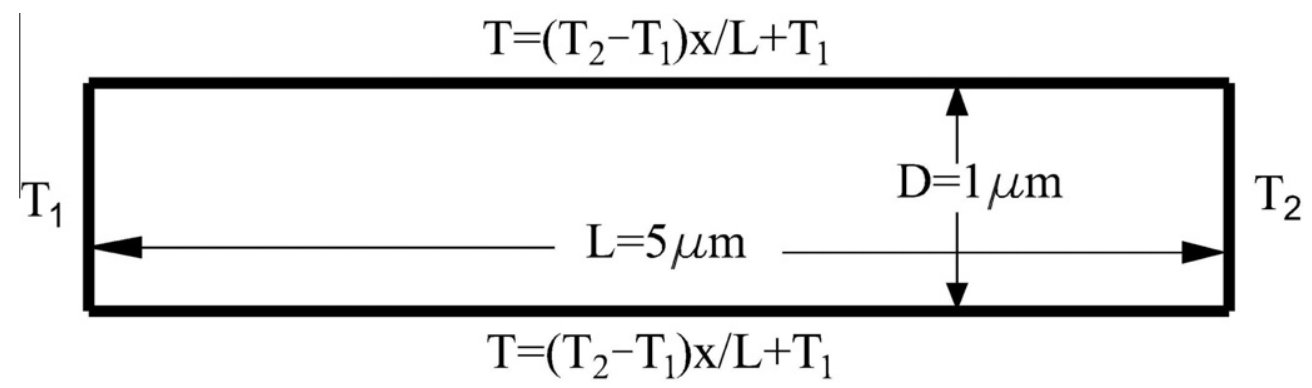

Fig. 9. Schematic diagram for the thermal creep flow.

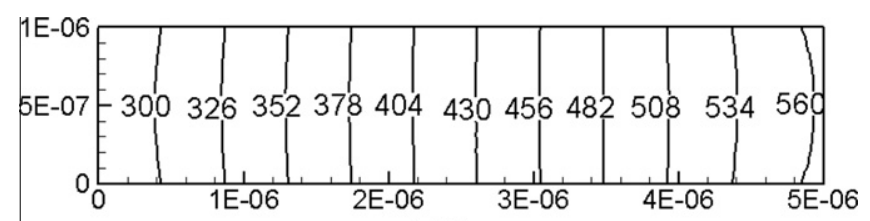

(a) IP

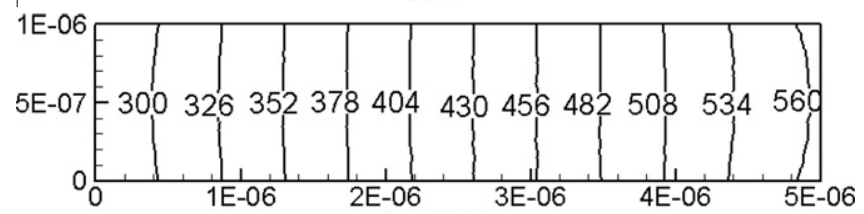

(b) DSMC

Fig. 10. Distribution of temperature contours for thermal creep flow: (a) IP; (b) DSMC.

$$
\frac{\left.\partial n \overline{\left(U_{y}^{2}+R T_{y y}\right)}\right)}{\partial t}=-\frac{\left.\partial 2 n R \overline{U_{y} T_{x y}}\right)}{\partial x}-\frac{\left.\partial 2 n R \overline{U_{y} T_{y y}}\right)}{\partial y} .
$$

Considering the nonequilibrium effect in the flow directions, flux splitting method is used to evaluate the update terms in Eqs. (39) and (40). According to Eq. (28), the expression of $\overline{T_{i j}}$ can be evaluated as follow: 


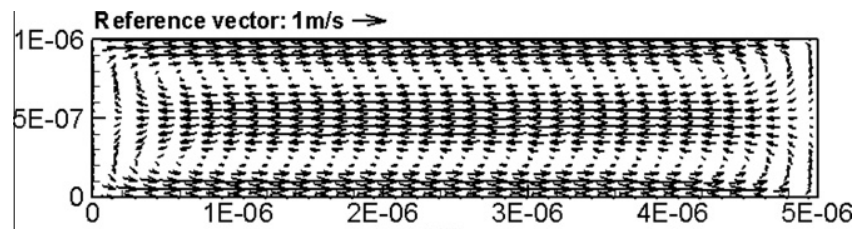

(a) IP

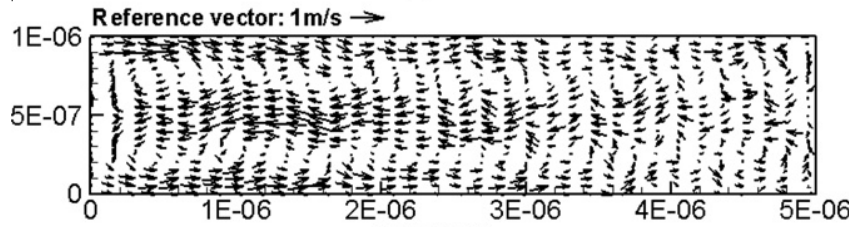

(b) DSMC

Fig. 11. Distribution of velocity vectors for thermal creep flow: (a) IP; (b) DSMC.

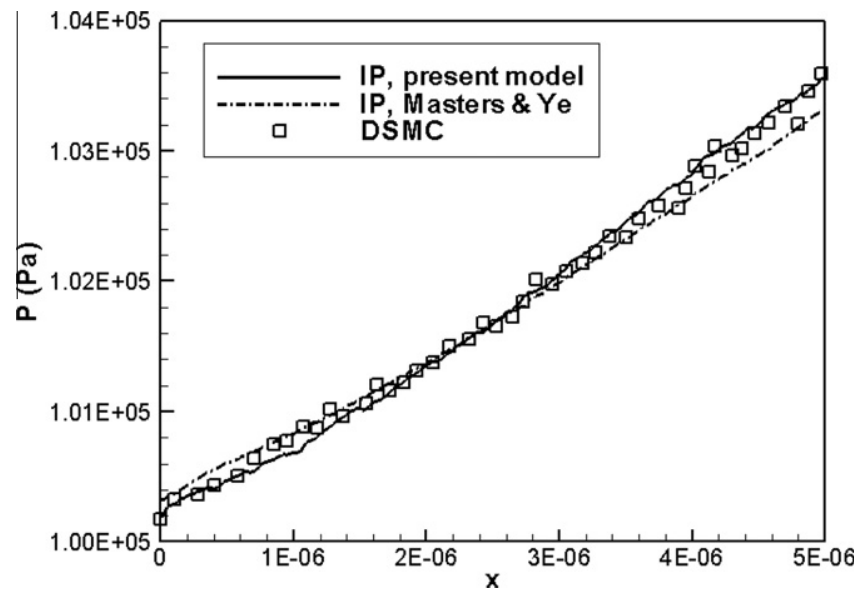

Fig. 12. Distribution of pressure along the $x$ direction at the midst of $y$ direction for thermal creep flow.

$$
\begin{aligned}
& \overline{T_{x x}}=\left.\overline{\frac{1}{4} T_{x x}}\right|_{\left(c_{x} \leqslant U_{x}, c_{y} \leqslant U_{y}\right)}+\left.\overline{\overline{1} T_{x x}}\right|_{\left(c_{x}>U_{x}, c_{y} \leqslant U_{y}\right)}+\left.\overline{\frac{1}{4} T_{x x}}\right|_{\left(c_{x} \leqslant U_{x}, c_{y}>U_{y}\right)}+\left.\overline{\overline{1}} T_{x x}\right|_{\left(c_{x}>U_{x}, c_{y}>U_{y}\right)}, \\
& \overline{T_{y y}}=\left.\overline{\frac{1}{4} T_{y y}}\right|_{\left(c_{x} \leqslant U_{x}, c_{y} \leqslant U_{y}\right)}+\left.\overline{\frac{1}{4} T_{y y}}\right|_{\left(c_{x}>U_{x}, c_{y} \leqslant U_{y}\right)}+\left.\overline{\frac{1}{4} T_{y y}}\right|_{\left(c_{x} \leqslant U_{x}, c_{y}>U_{y}\right)}+\left.\overline{\frac{1}{4} T_{y y}}\right|_{\left(c_{x}>U_{x}, c_{y}>U_{y}\right)}, \\
& \overline{T_{x y}}=\left.\frac{\overline{\sqrt{T_{x x} T_{y y}}}}{2 \pi}\right|_{\left(c_{x} \leqslant U_{x}, c_{y} \leqslant U_{y}\right)}-\left.\frac{\overline{\sqrt{T_{x x} T_{y y}}}}{2 \pi}\right|_{\left(c_{x}>U_{x}, c_{y} \leqslant U_{y}\right)}-\left.\frac{\overline{\sqrt{T_{x x} T_{y y}}}}{2 \pi}\right|_{\left(c_{x} \leqslant U_{x}, c_{y}>U_{y}\right)}+\left.\frac{\overline{\sqrt{T_{x x} T_{y y}}}}{2 \pi}\right|_{\left(c_{x}>U_{x}, c_{y}>U_{y}\right)} .
\end{aligned}
$$

For thermal creep flows, the shear stress $n R \overline{T_{x y}}$ is crucial to produce the creep mechanism. On the other hand, the transport of preserved temperature is mainly dependent on the molecular motions and collisions and is almost independent on the effect of update terms. Thus, the update terms in Eqs. (41) and (42) can be evaluated with the original expressions for simplification.

Fig. 10 shows the distribution of temperature contours in the field obtained by IP and DSMC simulations. The two results compare well with each other. Except for the regions close to the two end walls, the gas temperature almost follows the linear temperature distribution imposed by the horizontal walls. Different from the gas behavior in continuum regime, the distribution of velocity vectors in Fig. 11 shows that a pair of vortices is formed, with creep flow moving from cold to hot region along the horizontal walls and a central flow in the opposite direction. The sampling size of each computational cell for DSMC simulation is up to $1.7 \times 10^{7}$, while the corresponding sampling size for IP simulation is only $1.7 \times 10^{5}$. However, the statistical scatter of velocity in the IP result is much smaller than the DSMC result. Therefore, the total computation time needed for IP method is much less than that needed for DSMC method. Due to gas flow, the pressure in the field is no longer uniform. Fig. 12 presents the pressure distributions along the length of the microchannel at the midst of vertical direction. 
We compare the present IP result with the DSMC result and the published IP result obtained by Masters and Ye. All of them predict a static pressure gradient as expected.

\section{Conclusions}

In this paper, a model with multiple temperature components for the IP method is proposed. More important, we derive the governing equations for the IP quantities based on the Boltzmann equation by assuming each simulation molecule represents a Gaussian distribution function. According to the governing equations, the implementation of IP method is divided into molecular movement, molecular collision and update step. With a reasonable multiple temperature collision model and the flux splitting method for update, we successfully simulated the shear-driven Couette flow, external force-driven Poiseuille flow and thermal creep flow. All of the IP results have a good agreement with the corresponding DSMC results, and IP method uses much smaller sampling sizes than DSMC method. Therefore, it is believed that IP method is an accurate and efficient tool for simulating the nonequilibrium gas flows. A natural extension of this work is applying the multiple temperature model proposed here to other strongly nonequilibrium phenomena, such as the shock structure. Note that we have only considered monatomic molecule (Argon) in this paper. If polyatomic molecule is considered, and the flow temperature is high enough to excite the molecular rotational and vibrational degrees of freedom, the rotational and vibrational temperatures should be additionally preserved in simulation molecules as information to better capture the flow physics of strongly nonequilibrium flows. We recommend that further studies could be performed on this subject.

\section{Acknowledgments}

This work was supported by the National Natural Science Foundation of China (Grant Nos. 10921062 and 11002147).

\section{References}

[1] H. Struchtrup, Macroscopic Transport Equations for Rarefied Gas Flows, Springer-Verlag, Berlin, 2005.

[2] M.M. Mansour, F. Baras, A.L. Garcia, On the validity of hydrodynamics in plane Poiseuille flows, Physica A 240 (1997) 255-267.

[3] J. Zhang, J. Fan, F. Fei, Effects of convection and solid wall on the diffusion in microscale convection flows, Phys. Fluids 22 (2010) 122005

[4] G.E. Karniadakis, A. Beskok, Micro Flows: Fundamentals and Simulation, Springer-Verlag, Berlin, 2002.

[5] M.S. Ivanov, S.F. Gimelshein, Computational hypersonic rarefied flows, Annu. Rev. Fluid Mech. 30 (1998) 469-505.

[6] G.A. Bird, Molecular Gas Dynamics and Direct Simulation of Gas Flows, Clarendon, Oxford, 1994.

[7] E.S. Piekos, K.S. Breuer, Numerical modeling of micromechanical devices using the direct simulation Monte Carlo method, J. Fluids Eng.-Trans. ASME 118 (1996) 464-469.

[8] C.K. Oh, E.S. Oran, R.S. Sinkovits, Computations of high-speed, high Knudsen number microchannel flows, J. Thermophys. Heat Transfer 11 (1997) 497505.

[9] R.P. Nance, D.B. Hash, H.A. Hassan, Role of boundary conditions in Monte Carlo simulation of microelectromechanical systems, J. Thermophys. Heat Transfer 12 (1998) 447-449.

[10] E.S. Oran, C.K. Oh, B.Z. Cybyk, Direct simulation Monte Carlo: recent advances and applications, Annu. Rev. Fluid Mech. 30 (1998) $403-441$.

[11] C.R. Kaplan, E.S. Oran, Nonlinear filtering for low-velocity gaseous microflows, AIAA J. 40 (2002) 82-90.

[12] T.M.M. Homolle, N.G. Hadjiconstantinou, A low-variance deviational simulation Monte Carlo for the Boltzmann equation, J. Comput. Phys. 226 (2007) 2341-2358.

[13] T.M.M. Homolle, N.G. Hadjiconstantinou, Low-variance deviational simulation Monte Carlo, Phys. Fluids 19 (2007) 041701.

[14] J. Fan, C. Shen, Statistical simulation of low-speed unidirectional flows in transition regime, in: R. Brun (ed.), 21th International Symposium on Rarefied Gas Dynamics, Cepadus-Editions, Toulouse, 1999, pp. 245-252.

[15] J. Fan, C. Shen, Statistical simulation of low-speed rarefied gas flows, J. Comput. Phys. 167 (2001) 393-412.

[16] C.P. Cai, I.D. Boyd, J. Fan, G.V. Candler, Direct simulation methods for low-speed microchannel flows, J. Thermophys. Heat Transfer 14 (2000) $368-378$.

[17] C. Shen, J. Fan, C. Xie, Statistical simulation of rarefied gas flows in micro-channels, J. Comput. Phys. 189 (2003) 512-526.

[18] Q.H. Sun, I.D. Boyd, A direct simulation method for subsonic, microscale gas flows, J. Comput. Phys. 179 (2002) 400-425.

[19] Q.H. Sun, I.D. Boyd, Theoretical development of the information preserving method for strongly nonequilibrium gas flows, AIAA Paper (2005) 20054828.

[20] N.D. Masters, W.J. Ye, Octant flux splitting information preservation DSMC method for thermally driven flows, J. Comput. Phys. 226 (2007) $2044-2062$.

[21] J.C. Maxwell, On the dynamical theory of gases, Philos. Trans. Roy. Soc. London 157 (1867) 49-88.

[22] L.H. Holway, New statistical models for kinetic theory: methods of construction, Phys. Fluids 9 (1966) 1658-1673.

[23] C.D. Levermore, Moment closure hierarchies for kinetic theories, J. Stat. Phys. 83 (1996) 1021-1065.

[24] K. Xu, Z.L. Guo, Generalized gas dynamic equations with multiple translational temperatures, Mod. Phys. Lett. B 23 (2009) $237-240$.

[25] M. Tij, A. Santos, Perturbation analysis of a stationary nonequilibrium-flow generated by an external force, J. Stat. Phys. 76 (1994) $1399-1414$.

[26] K. Xu, H.W. Liu, J.Z. Jiang, Multiple-temperature kinetic model for continuum and near continuum flows, Phys. Fluids 19 (2007).

[27] K. Aoki, P. Degond, S. Takata, H. Yoshida, Diffusion models for Knudsen compressors, Phys. Fluids 19 (2007) 117103.

[28] T. Ohwada, Y. Sone, K. Aoki, Numerical analysis of the shear and thermal creep flows of a rarefied gas over a plane wall on the basis of the linearized Boltzmann-equation for hard-sphere molecules, Phys. Fluids A 1 (1989) 1588-1599.

[29] F. Sharipov, Non-isothermal gas flow through rectangular microchannels, J. Micromech. Microeng. 9 (1999) 394-401.

[30] J.R. Bielenberg, H. Brenner, A continuum model of thermal transpiration, J. Fluid Mech. 546 (2006) 1-23.

[31] D.H. Papadopoulos, D.E. Rosner, Enclosure gas flows driven by nonisothermal walls, Phys. Fluids 7 (1995) 2535-2537. 